\title{
Experiencia de innovación docente a partir de la aplicación de un ciclo de mejora en Geomorfología estructural
}

\author{
CÉSAR BORJA BARRERA \\ Universidad de Sevilla \\ Departamento de Geografía Física y A.G.R \\ cesarborja@us.es \\ ORCID: https://orcid.org/0000-0002-2157-7250 \\ D.O.I.: http://dx.doi.org/10.12795/JDU.2018.i01.96 \\ Pp.: $1707-1723$
}

\section{Resumen}

El presente trabajo recoge los resultados de la aplicación de un ciclo de innovación docente al bloque temático de Geomorfología Estructural, dentro de la asignatura Geomorfología e Hidrología. Se pretende con este CMD emprender una transición progresiva desde una enseñanza tradicional y transmisiva, hacia una nueva orientación más integradora, en la que el alumno adquiera un mayor protagonismo. Se utilizan preguntas clave al comienzo de cada sesión con las que discutir y construir los contenidos, habiendo sido dichas preguntas utilizadas, además, como cuestionario inicial (preevaluación) de cara a valorar el nivel de conocimiento de los alumnos, y, posteriormente, como cuestionario final (posevaluación) a fin de establecer el grado de aprendizaje alcanzado. Finalmente, y con el objetivo de determinar el nivel de conocimiento adquirido por los estudiantes, se han realizado 
diversas escaleras de aprendizaje que han mostrado unos resultados no demasiado satisfactorios, pero que señalan el camino a seguir en el futuro, aunque con la incorporación de mejoras oportunas.

Palabras claves: Geomorfología e Hidrología, Grado de Geografía y Gestión del Territorio, Docencia universitaria e experimentación docente universitaria, Geomorfología estructural

\section{Contexto de la intervención}

El Ciclo de Mejora Docente (CMD) implementado en la Fase de Permanencia de la REFID se lleva a cabo en el tema correspondiente a Geomorfología Estructural, dentro de la asignatura Geomorfología e Hidrología. Se trata de una asignatura obligatoria que se imparte de forma compartida para alumnos de 2o Curso del Grado en Geografía y Gestión del Territorio y alumnos de 3o curso del Doble Grado en Geografía y Gestión del Territorio e Historia, a la que, eventualmente, se incorporan alumnos del programa Erasmus, así como estudiantes estadounidenses que realizan estancias en la Universidad de Sevilla en el marco del programa de los cursos concertados que oferta la Facultad de Geografía e Historia. La asignatura completa es impartida en su totalidad por el mismo profesor (el que suscribe el presente trabajo), a lo largo del primer cuatrimestre en los dos grupos existentes (mañana y tarde), los miércoles y jueves, en bloques de dos horas, de 12,00 a 14,00, en horario de mañana, y de 18,00 a 20,00, en horario de tarde. Se trata de una asignatura de 6 créditos, repartidos en 4,5 teórico-prácticos (3 de teoría y 1,5 de prácticas) y 1,5 de seminario. Constituye una materia que procede de la fusión de dos asignaturas independientes y, hasta cierto punto, yuxtapuestas, que la última reforma del Plan de Estudios de Geografía convirtió en una sola de duración cuatrimestral. El presente ciclo de mejora docente se desarrollará a lo largo de 12 horas presenciales de clase, repartidas en 9 horas dedicadas a contenidos teóricos y 3 horas a contenidos prácticos. 
El objetivo principal de la asignatura en su conjunto consiste en iniciar al alumno en el conocimiento y comprensión de los principios metodológicos y conceptuales básicos de la Geomorfología y la Hidrología, así como en el manejo de su terminología específica. Se pretende, por un lado, que el alumno reconozca y comprenda los procesos fundamentales que intervienen en la conformación del relieve y en el funcionamiento de los sistemas hidrológicos, y de las relaciones dinámicas que se establecen entre ellos, y, por otro, que identifique y entienda las dimensiones temporales y espaciales propias de los hechos hidro-geomorfológicos.

\section{Diseño previo del Ciclo de Mejora Docente}

\section{Mapa de contenidos}

El bloque temático trabajado en el CMD corresponde, como ya se ha indicado, al tema 2 completo (teoría y práctica) correspondiente a la asignatura Geomorfología Estructural, cuyo objeto de estudio fundamental son los relieves estructurales (Gutiérrez Elorza, 2008; Pedraza, 1999). En la figura 1 se muestran de forma relacionada los principales contenidos teóricos y prácticos que se van a desarrollar en el CMD.

Jornadas de Formación e Innovación Docente del Profesorado | № 1 (2018) Esta obra se distribuye con la licencia Creative Commons 


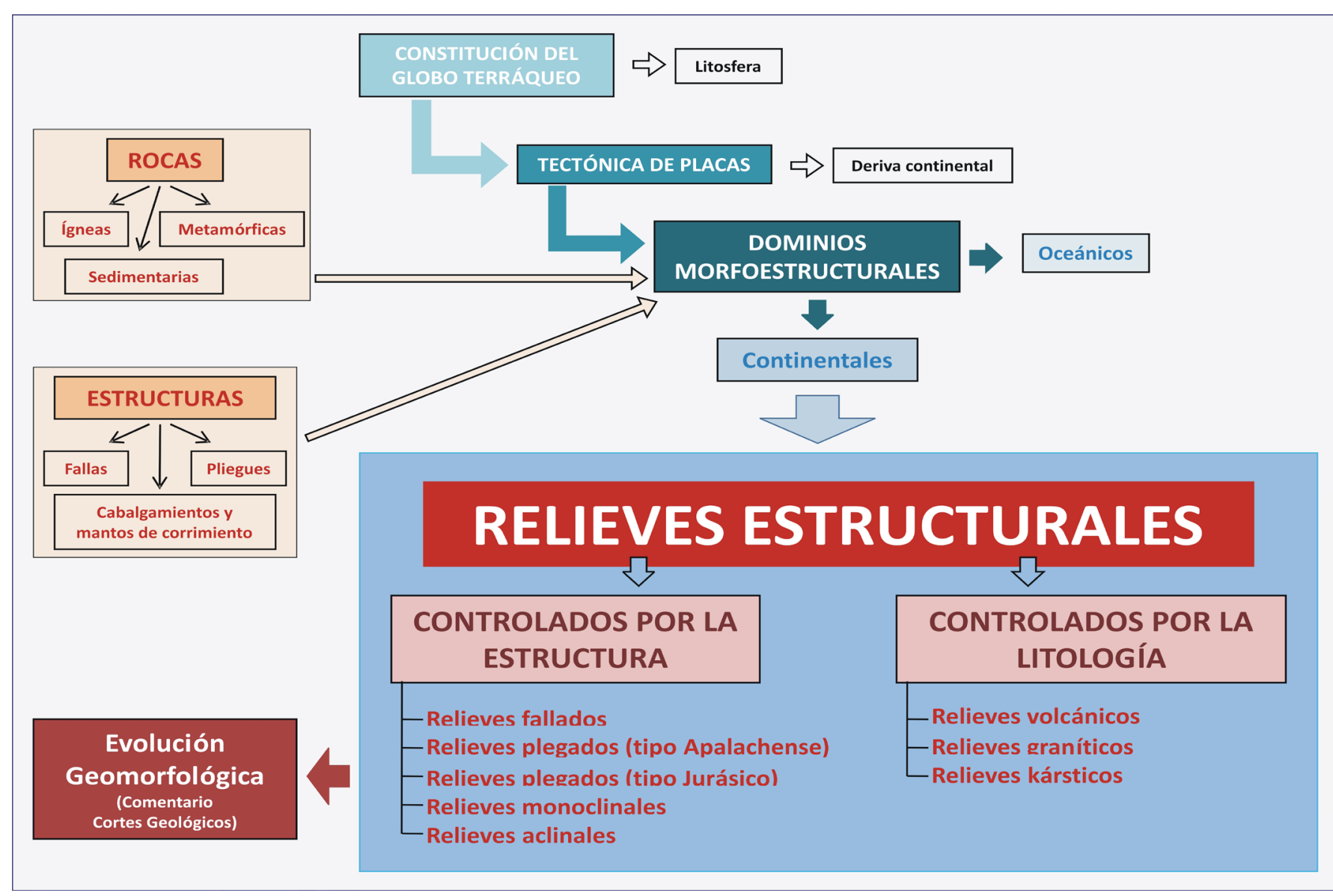

Figura 1. Mapa de contenidos.

Jornadas de Formación e Innovación Docente del Profesorado | № 1 (2018)

cc (†) Esta obra se distribuye con la licencia Creative Commons 


\section{Modelo metodológico y Secuencia de Actividades}

Se pretende en esta nueva fase de permanencia en la REFID la transición progresiva desde una forma de entender la enseñanza llevada a cabo hasta ahora, de corte más tradicional y transmisiva, y que no consideraba esencial las ideas previas de los alumnos, hacia una nueva orientación más integradora, basada en la consideración de las ideas o modelos mentales previos de los alumnos (Borja, 2017). Se pretende generar un entorno de aprendizaje más acorde con las premisas indicadas, en el que la figura del profesor vaya perdiendo cada vez más peso en el proceso de aprendizaje y los alumnos adopten un rol más activo y dinámico coincidiendo con las ideas de Finkel (2008). En esta línea, se aplicarán a lo largo del CMD algunas técnicas que propicien este nuevo enfoque, como son, por un lado, el planteamiento de preguntas clave al inicio de los bloques de contenidos que al final de cada sesión se discutirán, y, por otro, la realización de cuestionarios como herramienta para conocer las ideas previas de los alumnos para, a partir de ahí, construir un discurso y organizar unos contenidos adaptados a la realidad del aula (Bain, 2006).

Como se ha comentado, se pretende ir pasando progresivamente de un modelo metodológico de carácter transmisivo, a uno más dinámico, en el que la participación del alumno sea más activa y, sobre todo, más continuada, tal como se refleja en la figura 2 (modelo ideal/ posible). En este sentido, la dinámica de las clases pasará, en primer lugar, por el planteamiento de las preguntas clave, el debate y la aportación de los alumnos, así como, por la exposición por parte del profesor de los contenidos relacionados con las preguntas clave, utilizando para ello presentaciones power point en las que se insertarán de forma genérica, tanto textos extractados de los manuales de la asignatura, como vídeos, fotografías, material gráfico y cartográfico... Con todos los materiales aportados se 
discutirá y concretarán las ideas (repuestas a las preguntas iniciales) al final de cada sesión.

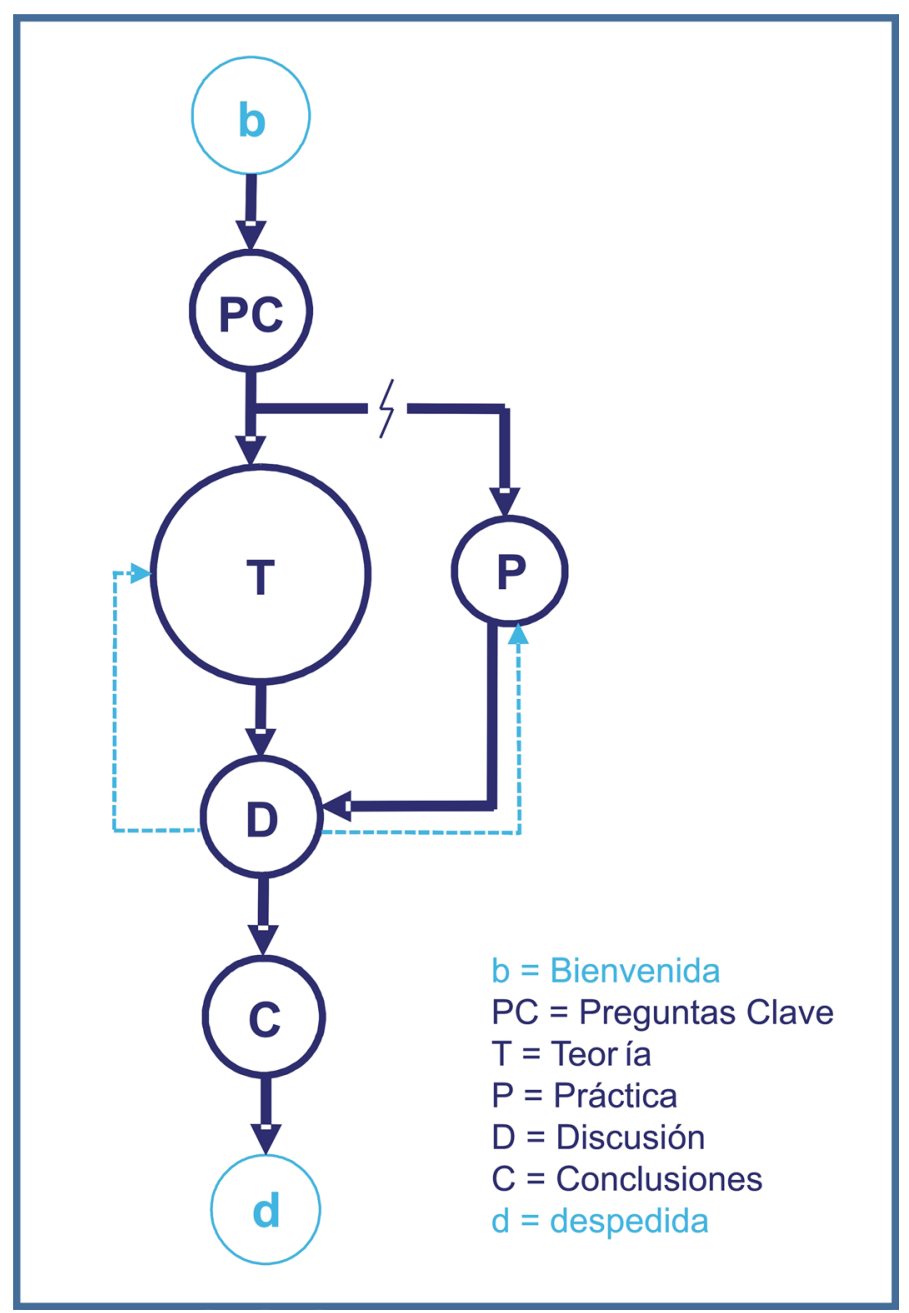

Figura 2. Modelo metodológico posible / ideal.

En la Tabla 1 se recoge la secuencia de actividades detallada propuesta para el desarrollo del bloque temático de Geomorfología Estructural.

Jornadas de Formación e Innovación Docente del Profesorado | № 1 (2018)

(c) Esta obra se distribuye con la licencia Creative Commons Reconocimiento-NoComercial-SinObraDerivada $\quad 4.0$ Internacional (CC BY-NC-ND 4.0.) 
Tabla 1. Secuencia de actividades.

\begin{tabular}{|c|c|c|c|c|c|c|c|}
\hline Sesión & $\begin{array}{c}\text { Saludo Inicial/ } \\
\text { bienvenida }\end{array}$ & $\begin{array}{l}\text { Planteamientos } \\
\text { generales } \\
\text { previos o finales }\end{array}$ & $\begin{array}{l}\text { Cuestiones } \\
\text { clave }\end{array}$ & Contenidos & $\begin{array}{c}\text { Recopilación } \\
\text { ideas }\end{array}$ & Conclusiones & Despedida \\
\hline $1 \underline{a}$ & \multirow{2}{*}{ 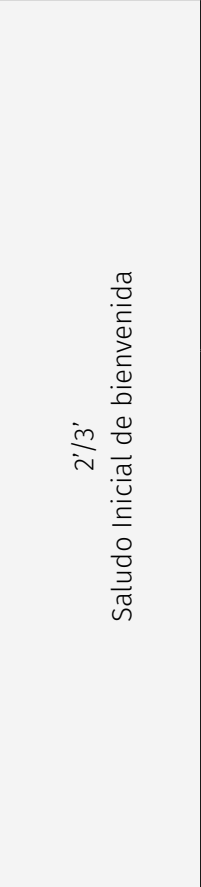 } & $\begin{array}{l}\text { 10' Presentación ge- } \\
\text { neral del CMD } \\
30^{\prime} \text { Cuestiona- } \\
\text { rio inicial com- } \\
\text { pleto sobre el CMD } \\
\text { en su totalidad } \\
\text { (Preguntas Clave) } \\
\text { (PREEVALUACIÓN) }\end{array}$ & $\begin{array}{l}\text { 10' Preguntas clave } \\
\text { 1. ¿Qué es la litosfera? } \\
\text { 2. ¿Qué elementos la com- } \\
\text { ponen? 3. ¿Cuáles son los } \\
\text { fundamentos de la Teoría } \\
\text { de la Tectónica de Placas? } \\
\text { 4. ¿Cuáles son sus principa- } \\
\text { les características y efectos } \\
\text { en la superficie terrestre? }\end{array}$ & \begin{tabular}{|l|}
50 \\
teóricos Contenidos \\
La constitución \\
del Globo \\
La tectónica de \\
placas
\end{tabular} & \multirow{2}{*}{ 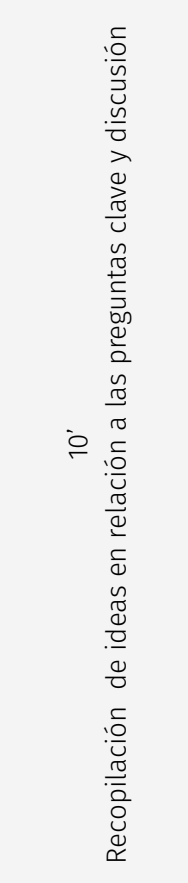 } & \multirow[b]{2}{*}{ 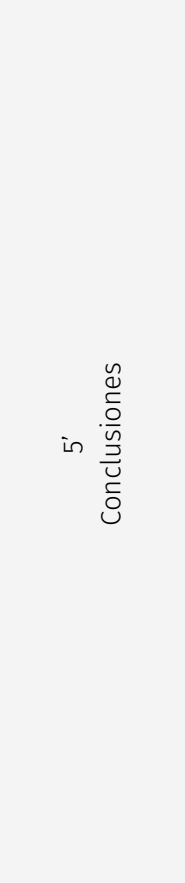 } & \multirow[b]{2}{*}{$\frac{\substack{\frac{\pi}{0} \\
\frac{0}{0}}}{\stackrel{0}{0}}$} \\
\hline 2 a & & & $\begin{array}{l}\text { 10' Preguntas clave } \\
\text { 5. ¿Qué elementos definen } \\
\text { un dominio morfoestruc- } \\
\text { tural? } \mathbf{6} \text {. ¿Cuántos tipos de } \\
\text { dominios morfoestructura- } \\
\text { les existen? 7. ¿Qué es una } \\
\text { roca? 8. ¿Cuántos tipos bá- } \\
\text { sicos hay? 9. ¿En qué se di- } \\
\text { ferencian unas de otras? } \\
\text { 10. ¿Qué es el análisis es- } \\
\text { truc-tural? 11. ¿En qué se } \\
\text { basa? 12. ¿Cuáles son los } \\
\text { principales tipos de estruc- } \\
\text { turas que existen? }\end{array}$ & \begin{tabular}{|l|}
$90^{\prime}$ Contenidos \\
teóricos. \\
Los dominios \\
morfoestruc-tu- \\
rales \\
Las rocas \\
El análisis \\
estructural
\end{tabular} & & & \\
\hline
\end{tabular}

Jornadas de Formación e Innovación Docente del Profesorado | № 1 (2018)

(c) (7) E $($ Esta obra se distribuye con la licencia Creative Commons

(c) $)(\Theta)$ Reconocimiento-NoComercial-SinObraDerivada 


\begin{tabular}{|c|c|c|c|}
\hline $3 \underline{a}$ & & $\begin{array}{l}\text { 10' Preguntas clave } \\
\text { 13. ¿Qué elementos con- } \\
\text { dicionan los relieves es- } \\
\text { tructurales? } \mathbf{1 4} \text {. ¿Cuántos } \\
\text { tipos de relieves estructura- } \\
\text { les controlados por la dis- } \\
\text { posición tectónica hay? } \mathbf{1 5 .} \\
\text { ¿Cuáles son las principales } \\
\text { diferencias entre ellos? }\end{array}$ & $\begin{array}{l}90 \text { ' Contenidos } \\
\text { teóricos } \\
\text { Relieves estruc- } \\
\text { turales con- } \\
\text { trolados por la } \\
\text { tectónica }\end{array}$ \\
\hline $4 \underline{a}$ & & $\begin{array}{l}\text { 10' Preguntas clave } \\
\text { 16. ¿Cuántos tipos de relie- } \\
\text { ves estructurales controla- } \\
\text { dos por la litología hay? } 17 . \\
\text { ¿Cuáles son las principales } \\
\text { diferencias entre ellos? }\end{array}$ & $\begin{array}{l}90{ }^{\prime} \text { Contenidos } \\
\text { teóricos } \\
\text { Relieves estruc- } \\
\text { turales con- } \\
\text { trolados por la } \\
\text { litología. } \\
\end{array}$ \\
\hline $5 \underline{a}$ & & $\begin{array}{l}\text { 10' Preguntas clave } \\
\text { 18. ¿Cómo se puede aplicar } \\
\text { a la resolución de proble- } \\
\text { mas prácticos el conoci- } \\
\text { miento sobre los relieves } \\
\text { estructurales? }\end{array}$ & $\begin{array}{l}90^{\prime} \text { Aplicación } \\
\text { práctica de los } \\
\text { contenidos } \\
\text { Comentario } \\
\text { geomorfoló- } \\
\text { gico del corte } \\
\text { geológico. } \\
\end{array}$ \\
\hline $6 \underline{a}$ & $\begin{array}{l}\text { 30' Evaluación post } \\
\text { CMD a partir de } \\
\text { la respuesta a del } \\
\text { Cuestionario Inicial } \\
\text { (POSEVALUA-CIÓN). } \\
\text { 10' Evaluación del } \\
\text { docente. }\end{array}$ & $\begin{array}{l}\text { 10' Preguntas clave } \\
\text { 19. ¿Qué beneficios propor- } \\
\text { ciona los conocimientos } \\
\text { sobre los relieves estruc- } \\
\text { turales en el desarrollo del } \\
\text { ejercicio profesional del } \\
\text { geógrafo? }\end{array}$ & $\begin{array}{l}50^{\prime} \text { Aplicación } \\
\text { práctica de los } \\
\text { contenidos } \\
\text { Comentario } \\
\text { geomorfoló- } \\
\text { gico del corte } \\
\text { geológico. }\end{array}$ \\
\hline
\end{tabular}

Jornadas de Formación e Innovación Docente del Profesorado | № 1 (2018)

(c) (1) Esta obra se distribuye con la licencia Creative Commons

(c) $(7) \Theta$ Reconocimiento-NoComercial-SinObraDerivada 
Cuestionario inicial-final de seguimiento de la evolución

El cuestionario inicial (preevaluación) pasado a los alumnos se compone de las cuestiones con las que se abrieron cada uno de los bloques de contenidos desarrollados en las distintas sesiones, sobre los que se planteaba el desarrollo teórico por parte del profesor, partiendo del nivel de los alumnos constatado previamente. Se trató de un cuestionario compuesto de 17 preguntas que se agrupan en 7 cuestiones. El mismo cuestionario se volvió a pasar a finales del CMD (posevaluación) únicamente a aquellos alumnos presentes en el aula que respondieron al inicial, con el objetivo de poder comprar el nivel de aprendizaje alcanzado. Las preguntas clave con las que se confeccionó el cuestionario están recogidas en la Tabla 1.

\section{Aplicación del CMD}

\section{Relato resumido de las sesiones}

Cuando se les explicó a los alumnos en la primera sesión que iban a participar en una nueva experiencia docente de carácter innovador, su reacción inicial fue de desasosiego e incertidumbre ante una nueva iniciativa de la que no sabían nada. Por lo general, los estudiantes no están acostumbrados a ser los protagonistas del proceso de aprendizaje y no les gusta abandonar su zona de confort (que prácticamente se reduce a ser un sujeto pasivo en el aula), por lo que cualquier novedad les produce inicialmente recelo. Cuando se les solita que contesten a un cuestionario con preguntas relacionadas con los contenidos que aún no se han desarrollado en el aula (Preevaluación), se pasa a una actitud abiertamente de rechazo. No es hasta que se les aclara que el cuestionario al que van a 
responder no tendrá repercusión alguna en su nota final, y que deben contestar en conciencia lo que realmente sepan de cada una de las cuestiones, que acceden a realizarlo.

A partir de esta primera actividad (innovadora para el alumnado), todas las sesiones se comenzaron con el planteamiento de las preguntas-problemas clave que iban a ser desarrolladas posteriormente. En esta línea, en las primeras clases los alumnos fueron poco participativos al principio, aunque al final del módulo, ciertamente, han sido muy pocos los alumnos (que habitualmente asisten a clase con interés), los que no han participado en algún momento. La dinámica propuesta contemplaba el desarrollo de los contenidos con los que poder contestar a las cuestiones planteadas al inicio de la sesión, a partir de las explicaciones del profesor sobre un power point ilustrado con textos explicativos y numeroso material gráfico (fotografías, gráficas, tablas...). Al hilo de las explicaciones se iba interpelando constantemente a los alumnos, resolviendo cuestiones particulares de la explicación o haciendo que propusieran ejemplos prácticos y reales de las distintas problemáticas tratadas. Se observó una alta participación en esta dinámica, al tiempo que se generó bastante debate y discusión sobre los planteamientos propuestos entre los propios alumnos, permaneciendo el profesor en un segundo plano, actuando únicamente de moderador. El número de participantes en estas dinámicas fue elevado, especialmente en las últimas sesiones del módulo.

Los debates planteados en el aula derivaban, en ocasiones, hacia cuestiones que no estaban contempladas en el mapa de contenidos presentado, por lo que se planteó la aplicación de una nueva técnica que no teníamos prevista, y que hemos denominado píldora informativa, consistente en un pequeño dossier de ampliación de algunos de los temas que se hubieran abordado en la sesión, pero de los que, por cuestiones de tiempo, no se podian 
desarrollar más. Se trata de un pequeño trabajo de recopilación de información básica que, de forma voluntaria, alguno de los alumnos se encargaba de ampliar y enviar al resto de compañeros, previo visto bueno del profesor.

La dinámica de clase planteada se mantuvo durante el desarrollo de todo el módulo hasta la última sesión en la que se pasó de nuevo el cuestionario (Posevaluación) con las mismas preguntas del cuestionario inicial, de cara a establecer una evaluación del aprendizaje llevado a cabo por los alumnos. Se pretende comparar, de esta forma, las respuestas del cuestionario inicial con el final para comprobar el nivel de aprendizaje alcanzado por los estudiantes.

\section{Evaluación del aprendizaje de los estudiantes}

La comparación de las respuestas obtenidas en el cuestionario inicial (Preevaluación) y el final (Posevaluación) nos permiten valorar el nivel de aprendizaje alcanzado por el conjunto de los alumnos a lo largo del desarrollo del módulo. Para ello, se ha optado por la construcción de escaleras de aprendizaje (Rivero y Porlán, 2017) definidas a partir de las respuestas proporcionadas por un total de 38 alumnos (76 cuestionarios) que han sido los que, estando presentes en al aula, estuvieron el primer y último día del módulo. A aquellos otros que faltaron uno u otro día no se les recogió el cuestionario por la imposibilidad de comparar las respuestas iniciales con las finales. Las escaleras construidas se basan en la capacidad del alumno para superar obstáculos del conocimiento, es decir, en la destreza del estudiante para organizar en sus respuestas los contenidos trabajados, la habilidad para relacionarlos entre sí y expresarlos con la terminología correcta. Tras la revisión de las repuestas ofrecidas por los alumnos se ha optado por una escalera de cuatro escalones, en los que la diferente altura de de cada uno de ellos refleja, así mismo, el 
nivel de dificultad que debían superar los estudiantes en sus respuestas para alcanzar una categoría u otra.

Se recogen a continuación los resultados de las escaleras de aprendizaje obtenidos únicamente de 2 de las 7 cuestiones planteadas, por razón de espacio. Las cuestiones mencionadas se corresponden, en primer lugar, con la definición de la litosfera y, en segundo término, con la caracterización de la teoría de la tectónica de placas. Se muestran aquí, por tanto, escaleras de aprendizaje para la Cuestión 1 (preguntas 1 y 2) y la 5 (preguntas 10 a 12), identificando los criterios de adecuación en las respuestas, así como, indicando los obstáculos a superar en cada uno de los niveles.

En las figuras 3 a 6 se muestran los resultados obtenidos para las cuestiones analizadas con indicación del porcentaje de respuestas proporcionadas por los alumnos en dos series correspondientes al cuestionario inicial (rojo) y el final (azul). En relación con la Cuestión 1 (Figs. 3 y 4) sorprende notablemente que más del $86 \%$ del alumnado no sepa definir qué es y qué elementos componen la litosfera, dejando la pregunta en blanco o contestando de forma errónea o incompleta. Tan sólo el 10\% de los estudiantes fueron capaces elaborar una definición correcta y relacionar los principales elementos que la componen. El cuestionario final (Serie Azul) muestra una mejora en los resultados del aprendizaje significativo, aunque sigue siendo verdaderamente alarmante que un 34\% de alumnos no hayan adquirido el aprendizaje necesario para abordar con solvencia este escalón de la escalera de aprendizaje. Por su parte, tan sólo un 13\% de los estudiantes logra superar el último obstáculo del conocimiento, organizando una respuesta en la que definen correctamente el concepto e identifican los principales elementos constituyentes, al tiempo que son capaces de interrelacionar cada uno de ellos con el resto de compontes que integran la estructura interna del planeta. 


\section{CUESTIÓN 1:}

\section{1. ¿Qué es la litosfera?}

2. ¿Qué elementos la componen?

Respuesta en la que genera un definición completa del concepto y se identifican los elementos que la componen

Respuesta en la que genera una definición mínima del concepto

Respuesta en blanco Respuesta errónea

Obstáculo: uso de

terminología adecuada

Obstáculo: uso de

terminología adecuada

e identificación de

componentes básicos
Respuesta en la que genera un definición del concepto y se identifican

los elementos que la

integran. Se caracterizan mínimamente cada uno de estos elementos y se interrelacionan con el resto de elementos que componen el interior de planeta

Obstáculo: uso de terminología adecuada

e interrelación de conceptos

Figura 3. Baremo utilizado y escalones de dificultad con indicación de los obstáculos utilizados en la escalera de aprendizaje de la Cuestión 1.

\section{CUESTIÓN 1: \\ 1. ¿Qué es la litosfera? \\ 2. ¿Qué elementos la componen?}

Serie Roja: Cuestionario inicial

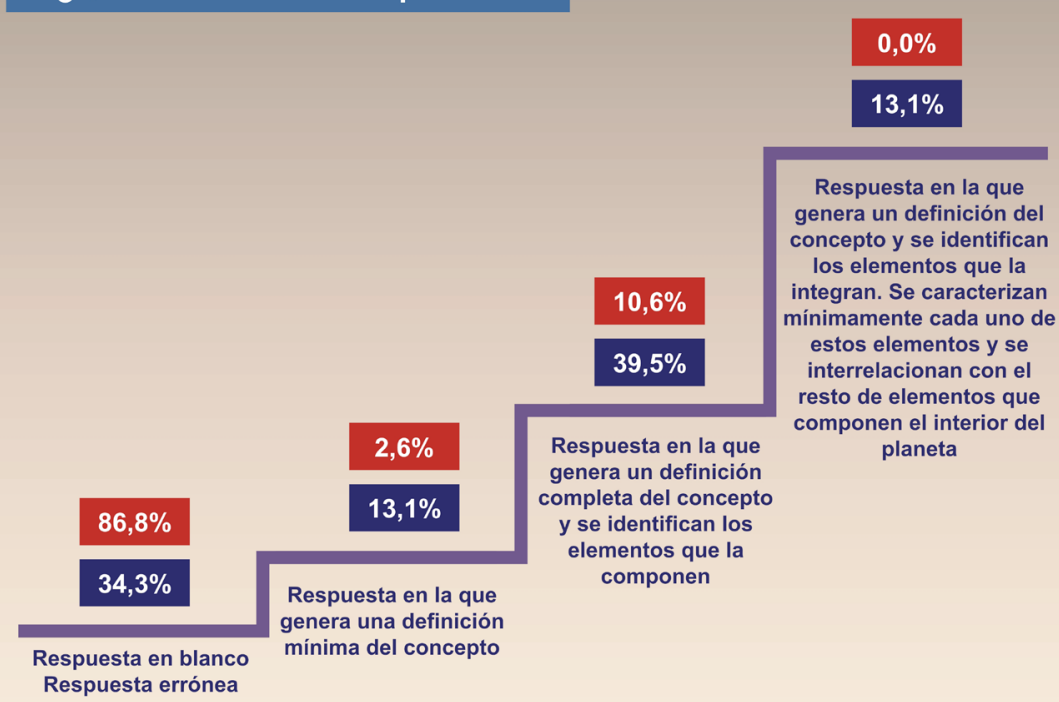

Figura 4. Escalera de aprendizaje con indicación de resultados del cuestionario inicial y final para la Cuestión 1. 
Los resultados obtenidos en las escaleras de aprendizaje de la Cuestión 5 (Figs. 5 y 6), no difieren mucho de los ya comentados para la Cuestión 1. Nuevamente el $86 \%$ de los estudiantes a la cuestión ¿qué es el análisis estructural?, contestaron de forma errónea o, directamente, la dejaron en blanco. Este porcentaje bajó posteriormente con el cuestionario final al 34\%, que sigue siendo un valor muy elevado. Es más llamativo todavía que tan solo un 5\% del alumnado fuera capaz de superar el último obstáculo del conocimiento y tuviera capacidad para definir y explicar correctamente el concepto de análisis estructural y relacionarlo con los distintos tipos de estructuras existentes en la superficie terrestre.

Los resultados expuestos para las cuestiones 1 y 5 muestran la tónica general evidenciada en el resto de las cuestiones que, por razón de espacio no mostramos. Sin embargo, en la Tabla 2 se recogen de forma resumida los porcentajes correspondientes a cada una de ellas.

\section{CUESTIÓN 5:}

10. ¿Qué es el análisis estructural?

11. ¿En qué se basa?

12. Cuáles son los principales tipos

de estructuras que existen?

Respuesta en la que se define el concepto de análisis estructural y se explica en qué se basa

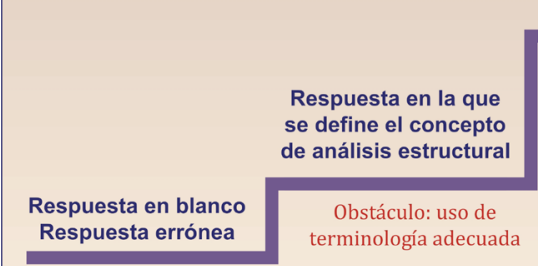

Figura 7. Baremo utilizado y escalones de dificultad con indicación de los obstáculos utilizados en la escalera de aprendizaje de la Cuestión 5.

Jornadas de Formación e Innovación Docente del Profesorado I № 1 (2018) Reconocimiento-NoComercial-SinObraDerivada Internacional (CC BY-NC-ND 4.0.) 


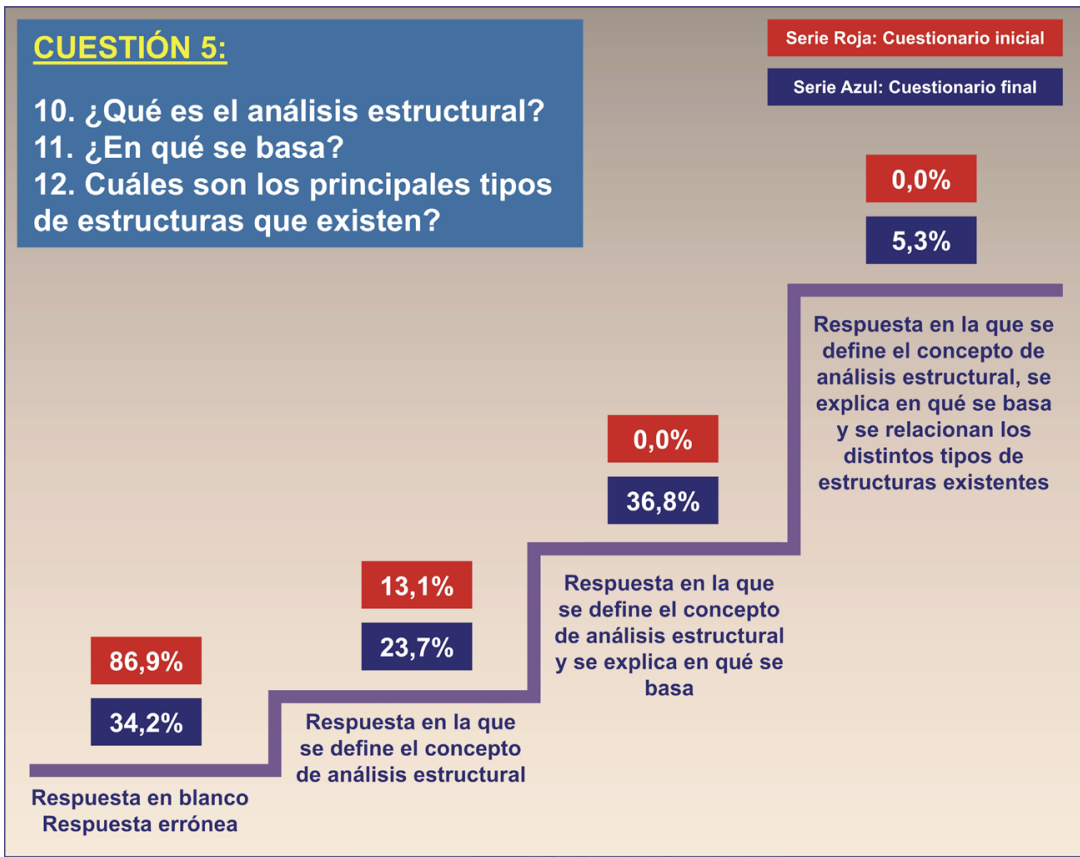

Figura 8. Escalera de aprendizaje con indicación de resultados del cuestionario inicial y final para la Cuestión 5.

Tabla 2. Síntesis de los resultados de las escaleras de aprendizaje

\begin{tabular}{|c|c|c|c|c|c|c|}
\hline \multirow{2}{*}{\multicolumn{2}{|c|}{ Cuestión }} & \multicolumn{5}{|c|}{ Nivel } \\
\hline & & & 1 & 2 & 3 & 4 \\
\hline \multirow{2}{*}{1} & \multirow{2}{*}{$\begin{array}{l}\text { 1. ¿Qué es la litosfera? } \\
\text { 2. ¿Qué elementos la } \\
\text { componen? }\end{array}$} & Pre & $86,6 \%$ & $2,6 \%$ & $10,6 \%$ & $0,0 \%$ \\
\hline & & Pos & $34,3 \%$ & $13,1 \%$ & $39,5 \%$ & $13,1 \%$ \\
\hline \multirow[b]{2}{*}{2} & \multirow{2}{*}{$\begin{array}{l}\text { 3. ¿Cuáles son los fundamentos } \\
\text { de la Teoría de la Tectónica de } \\
\text { Placas? } \\
\text { 4. ¿Cuáles son sus principales } \\
\text { características y efectos en la } \\
\text { superficie terrestre? }\end{array}$} & Pre & $26,3 \%$ & $26,3 \%$ & $39,5 \%$ & $7,9 \%$ \\
\hline & & Pos & $52,6 \%$ & $21,1 \%$ & $21,1 \%$ & 5,2 \\
\hline \multirow[b]{2}{*}{3} & \multirow{2}{*}{$\begin{array}{l}\text { 5. ¿Qué elementos definen un } \\
\text { dominio morfoestructural? } \\
\text { 6. ¿Cuántos tipos de dominios } \\
\text { morfoestructurales existen? }\end{array}$} & Pre & $97,4 \%$ & $0,0 \%$ & $2,6 \%$ & $0,0 \%$ \\
\hline & & Pos & $57,9 \%$ & $7,9 \%$ & $31,6 \%$ & $2,6 \%$ \\
\hline
\end{tabular}




\begin{tabular}{|c|c|c|c|c|c|c|}
\hline \multirow[b]{2}{*}{4} & \multirow{2}{*}{$\begin{array}{l}\text { 7. ¿Qué es una roca? } \\
\text { 8. ¿Cuántos tipos básicos hay? } \\
\text { 9. ¿En qué se diferencian unas } \\
\text { de otras? }\end{array}$} & Pre & $57,9 \%$ & $31,6 \%$ & $10,5 \%$ & $0,0 \%$ \\
\hline & & Pos & $10,5 \%$ & $31,6 \%$ & $55,3 \%$ & $2,6 \%$ \\
\hline \multirow[b]{2}{*}{5} & \multirow{2}{*}{$\begin{array}{l}\text { 10. ¿Qué es el análisis } \\
\text { estructural? } \\
\text { 11. ¿En qué se basa? } \\
\text { 12. ¿Cuáles son los principa- } \\
\text { les tipos de estructuras que } \\
\text { existen? }\end{array}$} & Pre & $86,9 \%$ & $13,1 \%$ & $0,0 \%$ & $0,0 \%$ \\
\hline & & Pos & $34,2 \%$ & $23,7 \%$ & $36,8 \%$ & $5,3 \%$ \\
\hline \multirow[b]{2}{*}{6} & \multirow{2}{*}{$\begin{array}{l}\text { 13. ¿Qué elementos condicionan } \\
\text { los relieves estructurales? } \\
\text { 14. ¿Cuántos tipos de relieves } \\
\text { estructurales controlados por la } \\
\text { disposición tectónica hay? } \\
\text { 15. ¿Cuáles son las principales } \\
\text { diferencias entre ellos? }\end{array}$} & Pre & $86,9 \%$ & $7,9 \%$ & $2,6 \%$ & $2,6 \%$ \\
\hline & & Pos & $52,6 \%$ & $7,9 \%$ & 7,9\% & 31,6 \\
\hline \multirow[b]{2}{*}{7} & \multirow{2}{*}{$\begin{array}{l}\text { 16. ¿Cuántos tipos de relieves } \\
\text { estructurales controlados por la } \\
\text { litología hay? } \\
\text { 17. ¿Cuáles son las principales } \\
\text { diferencias entre ellos? }\end{array}$} & Pre & $94,7 \%$ & $5,3 \%$ & $0,0 \%$ & $0,0 \%$ \\
\hline & & Pos & $47,4 \%$ & $7,9 \%$ & $5,2 \%$ & $39,5 \%$ \\
\hline
\end{tabular}

\section{Evaluación del CMD puesto en práctica}

En cuanto a las cuestiones a mantener y cambios a introducir para un futuro CMD más amplio sería necesario, lógicamente, ampliar el mapa de contenidos convenientemente $\mathrm{y}$, por tanto, elaborar nuevas preguntas clave. Del mismo modo, se podría avanzar más en un modelo ideal/real más integrador, para lo cual se podrían utilizar nuevas técnicas de aprendizaje (talleres, puestas en común, trabajo más autónomo del alumno -tipo píldoras informativas-, entre otras) de cara a "enganchar" a aquellos estudiantes que muestran escaso interés, e, incluso, a aquellos que estando motivados no alcanzan los objetivos de aprendizaje básicamente por no trabajar lo suficiente durante las sesiones. Se trataría, en última instancia, de intentar transformar al alumno en un sujeto más activo haciéndolo participar de forma más dinámica en el proceso de aprendizaje. En este sentido, la incorporación de 
técnicas como la realización de cuestionarios de preevaluación y posevaluación con las que identificar las ideas previas del alumnado y el nivel de aprendizaje alcanzado, constituirá a partir de este momento un elemento imprescindible en la práctica docente habitual. Este tipo de actividades podría, incluso, utilizarse como herramienta de evaluación en sustitución del clásico examen final.

\section{Referencias bibliográficas}

Bain, K. (2006). Lo que hacen los mejores profesores de universidad. Valencia: Universidad de Valencia.

Borja, C. (2017). "Experiencia de innovación docente universitaria en la enseñanza de la Geomorfología". En: IV Jornadas de Formación e Innovación Docente. Sevilla: Secretariado de Formación y Evaluación de la Universidad de Sevilla.

Finkel, D. (2008). Dar clase con la boca cerrada. Valencia: Universidad de Valencia.

Gutiérrez Elorza, M. (2008). Geomorfología. Madrid: Prentice Hall.

Pedraza, J. (1996). Geomorfología. Principios, métodos y aplicaciones. Madrid: Rueda.

Rivero, A. y Porlán, A. (2017). "La evaluación en la enseñanza universitaria". En: Enseñanza universitaria. Cómo mejorarla, R. Porlán (Coord.), Madrid: Morata.

Jornadas de Formación e Innovación Docente del Profesorado | № 1 (2018) Esta obra se distribuye con la licencia Creative Commons 